\title{
NONLINEAR DYNAMICS OF CARBON NANOTUBES UNDER LARGE ELECTROSTATIC FORCE
}

Tiantian $\mathrm{Xu}^{1}$

${ }^{1}$ Department of Mechanical Engineering, State University of New York at Binghamton, Binghamton, 13902 NY, USA, txu2@binghamton.edu

Mohammad I. Younis ${ }^{1,2}$

${ }^{1}$ Department of Mechanical Engineering, State University of New York at Binghamton, Binghamton, 13902 NY, USA, myounis@binghamton.edu

${ }^{2}$ Department of Mechanical Engineering, King Abdullah University of Science and Technology KAUST, Thuwal 23955-6900, Saudi Arabia, mohammad.younis@kaust.edu.sa

\section{Abstract}

Because of the inherent nonlinearities involving the behavior of CNTs when excited by electrostatic forces, modeling and simulating their behavior is challenging. The complicated form of the electrostatic force describing the interaction of their cylindrical shape, forming upper electrodes, to lower electrodes poises serious computational challenges. This presents an obstacle against applying and using several nonlinear dynamics tools typically used to analyze the behavior of complicated nonlinear systems undergoing large motion, such as shooting, continuation, and integrity analysis techniques. This works presents an attempt to resolve this issue. We present an investigation of the nonlinear dynamics of carbon nanotubes when actuated by large electrostatic forces. We study expanding the complicated form of the electrostatic force into enough number of terms of the Taylor series. Then, we utilize this form along with an Euler-Bernoulli beam model to study for the first time the dynamic behavior of CNTs when excited by large electrostatic force. The geometric nonlinearity and the nonlinear electrostatic force are considered. An efficient reduced-order model (ROM) based on the Galerkin method is developed and utilized to simulate the static and dynamic responses of the CNTs. Several results are generated demonstrating softening and hardening behavior of the CNTs near their primary and secondary resonances. The effects of the DC and AC voltage loads on the behavior have been studied. The impacts of the initial slack level and CNT diameter are also demonstrated.

\section{Introduction}

Nanoelectromechanical systems (NEMS) are a growing area of research, which extends the attractive features of the microelectromechanical systems (MEMS) technology to the nanoscale $[1,2]$. Currently NEMS are being investigated for a 
variety of applications in different fields, such as communications, health, and bioengineering, where they cover many different functions, including sensing and actuation. Their mechanical behavior analysis is a challenging task [3, 4]. Carbon nanotubes (CNTs), as one of the most promising kind of NEMS components, have been recently the subject of extensive research due to their unique electrical and mechanical properties. More in depth research however is needed for the mechanical response of CNTs to be able to implement them as functional devices and to utilize them as resonant sensors, switches, and actuators [5]. Particularly, extensive and accurate nonlinear dynamic analysis is needed to explain many of the reported experimental data and to reveal the full potential of CNTs when employed in device applications [6]. Beside their interesting mechanical properties, carbon nanotubes inherit their remarkable electrical properties from the unique electronic band structure of graphene. Electrically actuated CNTs were shown to have rich nonlinear behavior characterized by inherent nonlinear response even for small electric loads [7] as well as a wide range of tunability of their natural frequencies with the DC load [8].

Many investigations on the vibration of CNTs under several dynamic loadings have been conducted [1-19]. A nonlinear elastic beam model for an electrostatically excited nanotube resonator has been developed [9]. Based on this model, several particular nonlinear phenomena have been derived. So far, considerable research in this area has been concerned about the resonant frequency shift and the factors which lead to these shifts.

Most previous simulation studies model clamped-clamped CNTs to be perfectly straight. However, in practical fabrication process, fabricating perfectly straight CNTs with controlled geometry and orientation is difficult. Actually, clamped-clamped CNTs are usually fabricated with some level of curvature (slack) [10-15]. Thus, it is important to model the mechanics of initially slacked CNTs [10]. Experimental investigations by Sazonova et al. [11] and Sazonova [12] also show the importance of slack on estimating accurately the vibration behavior of CNTs.

Doubly clamped CNT resonators have been proven to be suitable as highly sensitive mass sensors and signal processing units, which have received much attention recently. Softening effect has been reported in single-walled carbon nanotube resonators [22]. Jin et al. [23] performed a study on the dynamic performance of CNT resonators taking into account the surface effect. It is found that the surface effect causes increased resonant frequency. Strong nonlinear coupling between mechanical modes in CNT resonators has been observed in [24]. They indicated that the strength of the mode coupling in carbon nanotubes is about six orders of 
magnitude larger than the mechanical-mode coupling in micromechanical resonators.

It is well known that the curvature of arches introduces quadratic and cubic nonlinearities. Furthermore, electrostatic forces are inherently nonlinear. The interaction of these nonlinearities (how they compete, strengthen, or cancel each other) still is not fully understood. Ouakad and Younis [25] investigated using a Galerkin procedure the nonlinear dynamic behavior of electrically actuated CNT resonators. They showed complex nonlinear dynamics phenomena, such as hysteresis, dynamic pull in, hardening and softening behaviors, and frequency bands with an inevitable escape from a potential well.

Ouakad and Younis [26,27] investigated in depth the nonlinearities of single-walled CNTs and their effects on the dynamics while varying the slack level and the DC voltages. The forced vibration response of CNTs under small electrodynamics actuation has been presented in [27] using perturbation techniques.

Because of the inherent nonlinearities involving the behavior of CNTs when excited by electrostatic forces, modeling and simulating their behavior is challenging. The complicated form of the electrostatic force describing the interaction of their cylindrical shape, forming upper electrodes, to lower electrodes poises serious computational challenges. This presents an obstacle against applying and using several nonlinear dynamics tools that commonly used to analyze the behavior of complicated nonlinear systems, such as shooting, continuation, and integrity analysis techniques. Hence, one objective of this work is to investigate an efficient model to simulate the nonlinear dynamic behavior of CNTs with different slack level under a wide range of voltage loads. Another objective is to present comprehensive simulations of the forced vibration response of CNTs under wide range of voltage loads using shooting techniques, which unlike the work in [27] is not limited to small voltage load or small motion. Such an investigation is presented for the first time. 


\section{Problem Formulation}

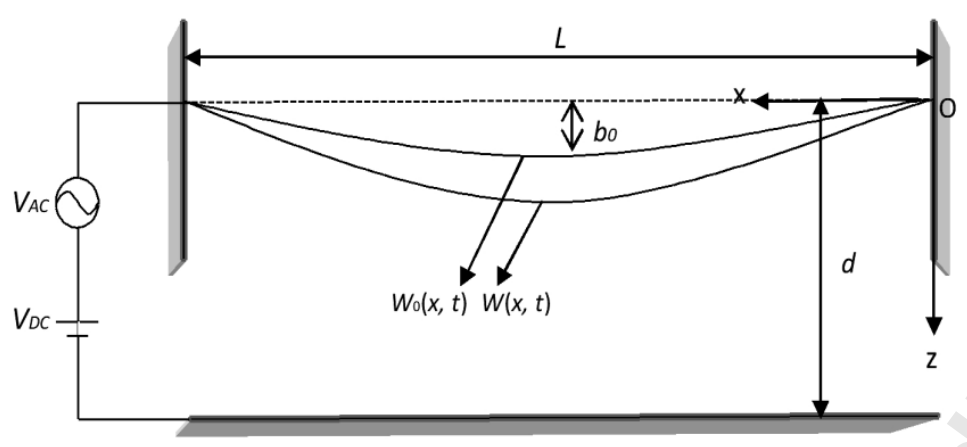

Fig.1 Schematic of an initially curved carbon nanotube.

To study the static and dynamic behavior of an electrically actuated slacked single-walled carbon nanotube, Fig. 1, we model it as a cylindrical Euler-Bernoulli beam of radius $\tilde{R}$, thickness $h$, and length $L$. The electrostatic force is introduced by an electrode underneath the nanotube with a gap width $d$. The nanotube is assumed to have a Young modulus $E$, a mass density $\rho$, a cross-sectional area $A$ and an area moment of inertia $I$. As mentioned before, the carbon nanotube is considered to be initially curved in the direction of the lower electrode with an initial shape $\hat{w}_{0}(\hat{x})=b_{0} \sin ^{2}\left(\frac{\pi \hat{x}}{L}\right)$

The governing equation describing the deflection $\hat{w}(\hat{x}, \hat{t})$ of the clamped-clamped CNT under electrostatic force can be written as $[6,9]$

$$
E I \frac{\partial^{4} \hat{w}}{\partial \hat{x}^{4}}+\rho A \frac{\partial^{2} \hat{w}}{\partial \hat{t}^{2}}+\tilde{c} \frac{\partial \hat{w}}{\partial \hat{t}}=\frac{E A}{2 L}\left[\int_{0}^{L}\left\{\left(\frac{\partial \hat{w}}{\partial \hat{x}}\right)^{2}-2\left(\frac{\partial \hat{w}}{\partial \hat{x}}\right)\right\} d x\right] \times\left[\frac{\partial^{2} \hat{w}}{\partial \hat{x}^{2}}-\frac{d^{2} \hat{w}_{0}}{d \hat{x}^{2}}\right]+\hat{F}_{e}
$$

where $\hat{t}$ is time and $\tilde{c}$ is the viscous damping coefficient.

The electrostatic force $\hat{F}_{e}$ is expressed as [6]

$$
\hat{F}_{e}=\frac{\pi \varepsilon_{0}\left(V_{D C}+V_{A C} \cos (\tilde{\Omega} \hat{t})\right)^{2}}{\sqrt{\left(d-\hat{w}-\hat{w}_{0}\right)\left(d-\hat{w}-\hat{w}_{0}+2 \tilde{R}\right)\left(\cosh ^{-1}\left(1+\frac{d-\hat{w}-\hat{w}_{0}}{\tilde{R}}\right)\right)^{2}}}
$$

where $\varepsilon_{0}$ is the air permittivity.

The boundary conditions are: 


$$
\hat{w}(0, \hat{t})=0, \frac{\partial \hat{w}}{\partial \hat{x}}(0, \hat{t})=0, \hat{w}(L, \hat{t})=0, \frac{\partial \hat{w}}{\partial \hat{x}}(L, \hat{t})=0
$$

\subsection{Limitation of the model}

The derivation of the continuum mechanics beam theory of (6) is based on the following assumption $[6,18]$ :

$a_{c} / L<<1$

$d_{N T} / L<<1$

where $d_{N T}$ is the CNT diameter and $a_{c}$ is the width of the hexagonal carbon rings, which is estimated to be near $0.24 \mathrm{~nm}$.

Moreover, the initial curvature of the arch (the slack) needs to remain small to satisfy the shallow arch theory:

$b_{0} / L<1$

For the considered case studies of this paper, these conditions are satisfied.

\subsection{Nondimensionalization}

We consider the dimensionless variables

$$
w=\frac{\hat{w}}{d}, x=\frac{\hat{x}}{L}, t=\frac{\hat{t}}{T}
$$

where $T$ is a time constant defined by $T=\sqrt{\left(\rho A L^{4}\right) /(E I)}$. Now we substitute (4) into (1)-(3). The dimensionless equations of motion and associated boundary conditions of the considered clamped-clamped CNT become:

$$
\begin{aligned}
& \frac{\partial^{4} w}{\partial x^{4}}+\frac{\partial^{2} w}{\partial t^{2}}+c \frac{\partial w}{\partial t}=\alpha_{2} F e+\alpha_{1}\left[\int_{0}^{1}\left\{\left(\frac{\partial w}{\partial x}\right)^{2}-2\left(\frac{\partial w}{\partial x} \frac{d w_{0}}{d x}\right)\right\} d x\right] \times\left[\frac{\partial^{2} w}{\partial x^{2}}-\frac{d^{2} w_{0}}{d x^{2}}\right] \\
& w(0, t)=0, \frac{\partial w}{\partial x}(0, t)=0, w(1, t)=0, \frac{\partial w}{\partial x}(1, t)=0
\end{aligned}
$$

where 


$$
\begin{aligned}
& F_{e}=\frac{\left(V_{D C}+V_{A C} \cos (\Omega t)\right)^{2}}{\sqrt{\left(1-w-w_{0}\right)\left(1-w-w_{0}+2 R\right)\left(\cosh ^{-1}\left(1+\frac{1-w-w_{0}}{R}\right)\right)^{2}}} \\
& w_{0}(x)=\frac{b_{0}}{d} \sin ^{2}(\pi x) \\
& \alpha_{1}=\frac{A d^{2}}{2 I}, \quad \alpha_{2}=\frac{\pi \varepsilon_{0} L^{2}}{E I d^{2}}, \quad c=\frac{\tilde{c} L^{4}}{E I}, \quad \Omega=\frac{\tilde{\Omega}}{\sqrt{(E I) /\left(\rho A L^{4}\right)}}, \quad R=\frac{\tilde{R}}{d}
\end{aligned}
$$

\subsection{Taylor-Series Expansion}

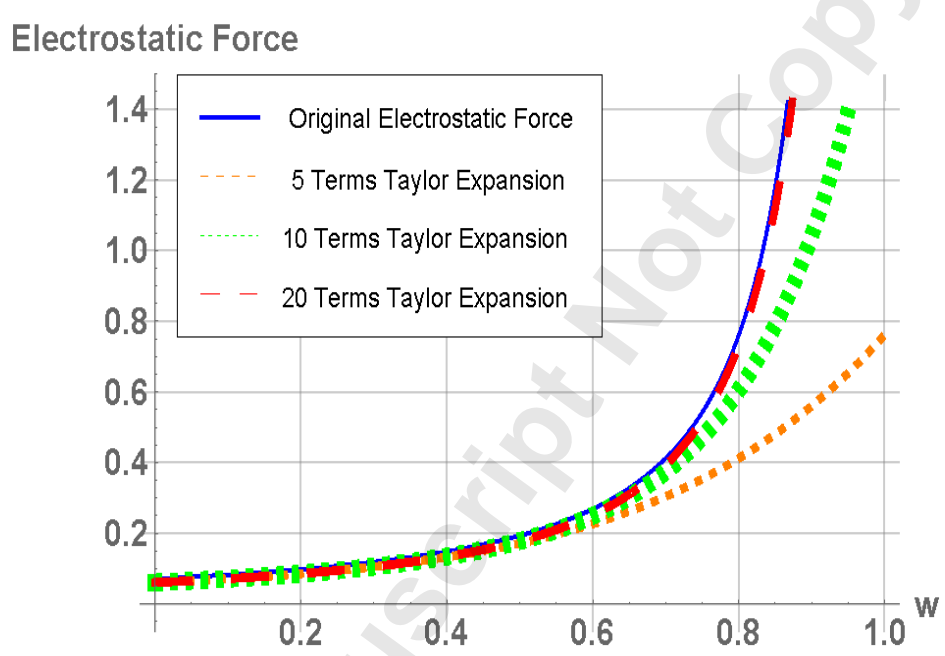

Fig.2 Comparison of the Taylor series expansion and the exact form of the nondimensional electrostatic force.

To reduce computational difficulty associated with the electrostatic force term in Eq. (7a) while applying the Galerkin procedure, we expand the complicated form of the electrostatic force into enough number of terms using Taylor series.

First of all, assume the main part of the electrostatic force a function of the deflection w:

$$
\begin{aligned}
& F_{e}=\left(V_{D C}+V_{A C} \cos (\Omega t)\right)^{2} F_{e_{-} m}(w) \\
& F_{e_{-} m}(w)=\frac{1}{\sqrt{\left(1-w-w_{0}\right)\left(1-w-w_{0}+2 R\right)\left(\cosh ^{-1}\left(1+\frac{1-w-w_{0}}{R}\right)\right)^{2}}}
\end{aligned}
$$

Then we expand this term into enough terms of Taylor series:

$$
F_{e} T_{n}(w)=a_{0}+a_{1} w+a_{2} w^{2}+a_{3} w^{3}+\cdots+a_{n} w^{n}
$$


To figure out how many terms expansion is accurate enough to replace the complicated force expression, we neglect the initial slack term $w_{0}$ in (9) here and get:

$$
F_{e 1_{-} m}(w)=\frac{1}{\sqrt{(1-w)(1-w+2 R)\left(\cosh ^{-1}\left(1+\frac{1-w}{R}\right)\right)^{2}}}
$$

$R$ here is normalized by the gap length and is constant.

Then we expand this in terms of $w$ :

$$
F_{e 1} T_{n}(w)=b_{0}+b_{1} w+b_{2} w^{2}+b_{3} w^{3}+\cdots+b_{n} w^{n}
$$

Where $b_{0}-b_{n}$ are the coefficients produced from the Taylor series expansion.

The deflection $w$ is normalized by $w=\frac{\hat{w}}{d}$ as mentioned in previous section. The range of $w$ should be from 0 to 1 since the deflection of the nanotube cannot exceed the gap length $d$.

Thus we could compare the expanded force term $F_{e 1} T_{n}(w)$ and the original one $F_{e 1 \_m}(w)$ as in Fig2. From Fig.2 we can see that by comparing the expanded form $F_{e 1} T_{n}(w)$ of the electrostatic force to the exact form $F_{e 1_{-} m}(w)$, at least twenty terms are needed to capture accurately the strong nonlinear form of the force over the full range of motion.

The percentage error between the Taylor series expressions and the original analytical expression of the electrostatics force can be expressed as

$$
E_{n}(w)=\frac{F_{e 1 \_m}(w)-F_{e 1} T_{n}(w)}{F_{e 1 \_m}(w)} \times 100 .
$$

The errors for different terms expansions are plotted in Fig. 3. 


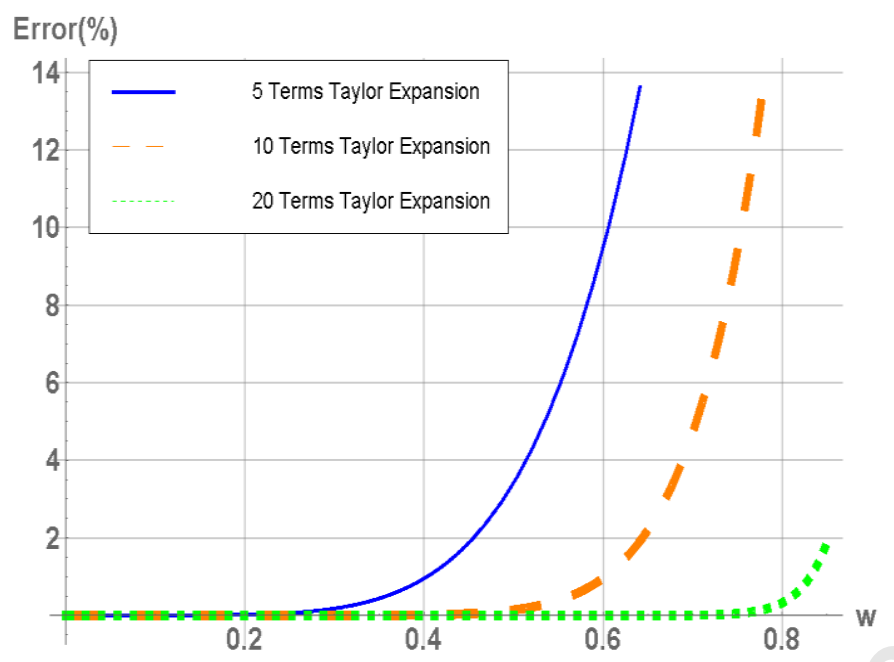

Fig.3 Percentage error of the Taylor series expansion of the nondimensional electrostatic force.

The deflection of the CNT cannot be higher than the gap length. We choose the range of $w$ from 0 to 0.85 to observe the error since when $w$ approaches 1 , the CNT approaches pull in, which is not the focus of this paper.

From Fig3 one can see that the error of the 20 terms Taylor expansion does not exceed $2 \%$, which is acceptable for all practical purposes. As a conclusion, 20 terms Taylor series is accurate enough to substitute the original electrostatic force term in the numerical computations.

Then we have:

$$
F_{e} T_{20}=b_{0}+b_{1} w+b_{2} w^{2}+b_{3} w^{3}+\cdots+b_{i} w^{i}+\cdots+b_{20} w^{20}
$$

Where, as before, $b_{0}, b_{1} \cdots \cdots b_{20}$ are the Taylor expansion coefficients.

Then we replace $F_{e}$ with $\left(V_{D C}+V_{A C} \cos (\Omega t)\right)^{2} F_{e} T_{20}(w)$ in (5) and get:

$$
\frac{\partial^{4} w}{\partial x^{4}}+\frac{\partial^{2} w}{\partial t^{2}}+c \frac{\partial w}{\partial t}=\alpha_{2}\left(V_{D C}+V_{A C} \cos (\Omega t)\right)^{2} F_{e} T_{20}(w)+\alpha_{1}\left[\int_{0}^{1}\left\{\left(\frac{\partial w}{\partial x}\right)^{2}-2\left(\frac{\partial w}{\partial x} \frac{d w_{0}}{d x}\right)\right\} d x\right] \times\left[\frac{\partial^{2} w}{\partial x^{2}}-\frac{d^{2} w_{0}}{d x^{2}}\right]
$$

It is worth to point out that one advantage of the Taylaor series expansion of the electrostatic force that it resolves the numerical problems reported in [27] when using a beam model, in which case a cable model had to be used. Here we do not face such a problem, and hence, we are able to keep the bending effect and use a beam model. 


\subsection{Galerkin Expansion}

To derive a reduced-order model ROM, we apply the Galerkin method and use the undamped unforced beam's linear orthonormal modeshapes $\phi_{i}(x)$ [25-27]. Hence the deflection is expressed as:

$$
w(x, t)=\sum_{i=1}^{n} u_{i}(t) \phi_{i}(x)
$$

where $u_{i}$ is the modal coordinate of the $i$ th mode. Following the standard procedure of Galerkin [20], nonlinear differential equations of $u_{i}(t)$ are derived, which can be integrated numerically to yield the dynamics response. As was indicated in [25], it is found that one mode is sufficient for convergence. In the absence of internal resonance, a one-mode approximation can yield adequate accuracy. However, the one mode approximation cannot capture the mode coupling effect or internal resonances, if any. This adds a limitation on our model. As an example for the resulting ROM, assuming one mode, the modal equation describing $u_{1}(t)$ for a clamped-clamped initially slacked CNT is written as:

$$
\begin{aligned}
& \ddot{u}_{1}(t)+c \dot{u}_{1}(t)+\kappa u_{1}(t)= \\
& \alpha_{1} \gamma u_{1}(t)^{3}+\alpha_{1} \eta u_{1}(t)^{2}+\alpha_{1} \beta u_{1}(t)+\alpha_{2}\left(V_{D C}+V_{A C} \cos (\Omega t)\right)^{2} \int_{0}^{1} F_{e} T_{20}\left(u_{1}, \phi_{1}\right) \phi_{1}(x) d x
\end{aligned}
$$

where

$$
\begin{aligned}
& \kappa=\int_{0}^{1} \phi_{1}(x) \phi_{1}^{\prime \prime \prime \prime}(x) d x, \\
& \gamma=\int_{0}^{1} \phi_{1}^{\prime}(x)^{2} d x \int_{0}^{1} \phi_{1}(x) \phi_{1}^{\prime \prime}(x) d x, \\
& \eta=-\left(\int_{0}^{1} \phi_{1}^{\prime}(x)^{2} d x \int_{0}^{1} \phi_{1}(x) w_{0}^{\prime \prime}(x) d x+\int_{0}^{1} \phi_{1}^{\prime}(x) w_{0}^{\prime}(x) d x \int_{0}^{1} \phi_{1}(x) \phi_{1}^{\prime \prime}(x) d x\right), \\
& \beta=2 \int_{0}^{1} \phi_{1}^{\prime}(x) w_{0}^{\prime}(x) d x \int_{0}^{1} \phi_{1}(x) w_{0}^{\prime \prime}(x) d x
\end{aligned}
$$




\section{Long Time Integration Results}

As a case study, a non-hollow CNT of $L=1000 \mathrm{~nm}, \tilde{R}=5 \mathrm{~nm}, d=200 \mathrm{~nm}$, Young modulus $E=1 \mathrm{TPa}$, and mass density $\rho=1.3 \mathrm{~g} / \mathrm{cm}^{3}$ is considered.

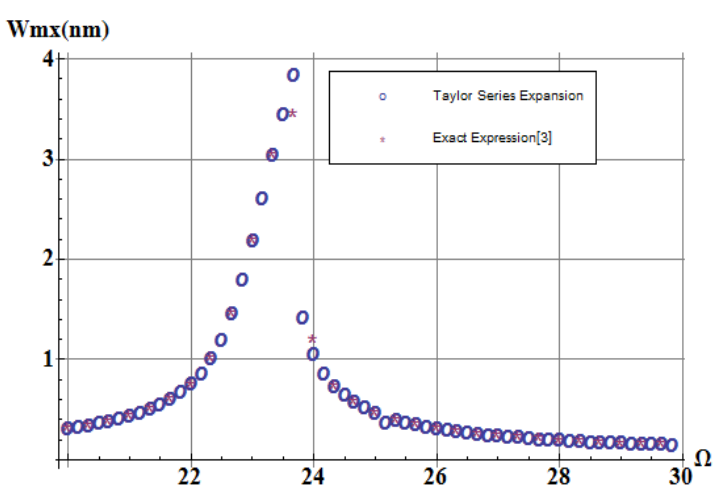

a

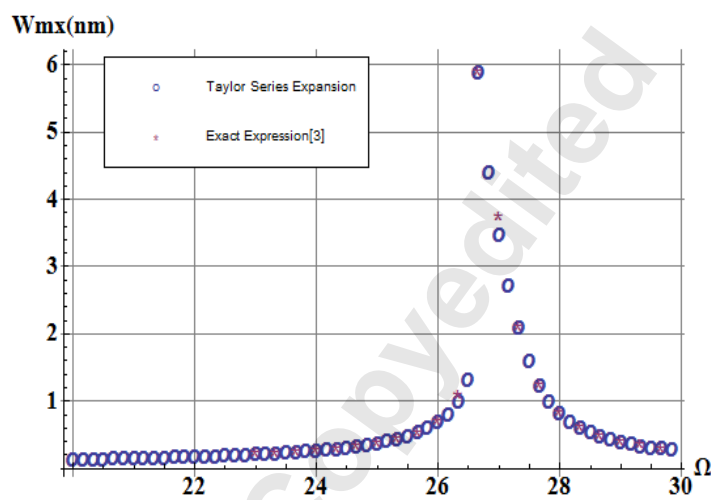

b

Fig. 4 Frequency-response curves of (a) $2 \mathrm{~nm}$ and (b) $5 \mathrm{~nm}$ slacked CNT showing hardening and softening behaviors, respectively. Results are shown for $V_{\mathrm{DC}}=V_{\mathrm{AC}}=1 \mathrm{~V}$ and a quality factor of 100 .

Fig.4 shows frequency response curves of two CNTs of two various slack levels. Numerical response with the same parameter calculated without the Taylor expansion from previous study of [27] are plotted in the same figure with numerical results using the simplified governing equation with the electrostatic force term substituted as its Taylor series. It has been mentioned in [27] that to get this result is quite computationally challenging and time consuming due to the spatial integration involving the complex electrostatic force term at each time step. Using the Taylor expansion alleviates this problem and enables efficient computation of the results. For the case of small slack as shown in Fig.4 (a), a hardening behavior occurs and then it switches to softening type behavior when the slack level increases to $5 \mathrm{~nm}$ as shown in Fig.4 (b).

\section{The Shooting Method}

The long-time integration technique may not predict accurately the dynamical behavior, especially near bifurcation points and under low damping conditions. Toward this, we use the shooting method [20]. The shooting method is a powerful technique for capturing periodic motion and analyzing their stability for nonlinear systems. Hence, it is capable of predicting both stable and unstable solutions. In this section, we utilize the shooting method in conjunction with the Floquet theory to obtain both stable and unstable solution of the frequency response of the CNT excited 
by electrostatic force.

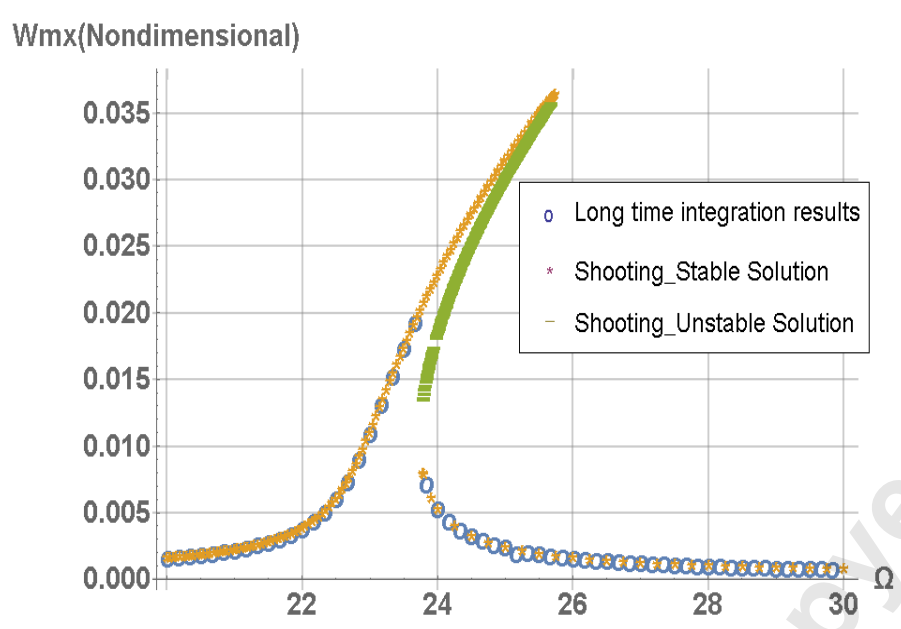

Fig.5. Frequency response curve of $2 \mathrm{~nm}$ slacked CNT.

Shown for $V_{\mathrm{DC}}=V_{\mathrm{AC}}=1 \mathrm{~V}$ and a quality factor of 100 .

We consider the same case study of last section. First we study the advantage of the shooting method on revealing the nonlinear behavior of CNT under a certain electrostatic force. Fig.5 shows that the shooting method result and longtime integration method has a good match. Moreover, the shooting method can predict not only unstable solutions, which longtime integration method cannot get, but it can show the entire range of the stable solutions. Also the Floquet multipliers are monitored during the calculation process. The absolute values of the Floquet multipliers of both stable and unstable solutions are getting extremely close to 1 when the stable solution and unstable solution meets indicating a tangent bifurcation.

\section{Results and Discussions}

Next we use the shooting technique to obtain results for a wide range of voltage loads. Fig.6 shows the response of a 5nm slacked CNT under a voltage load of $V_{D C}=V_{A C}=1 \mathrm{~V}$. One can see a complicated nonlinear behavior, in which the CNT shows a softening behavior for low amplitude of vibration, after which it turns into a hardening behavior, as seen by the new stable branch that is created above $6 \mathrm{~nm}$ amplitude. The local softening behavior has been predicted in [9], for the same case parameters, which indicates that for a slack level $b_{0}>4 n m$, the quadratic nonlinearities due to the initial curvature and electrostatic force dominate the dynamic behavior of the slacked CNT leading to the softening effect. However, since the model in [9] is based on perturbation analysis that is local and valid for small 
amplitude of motion, the emergence of the new stable branch with the hardening behavior has not been captured by the perturbation model.

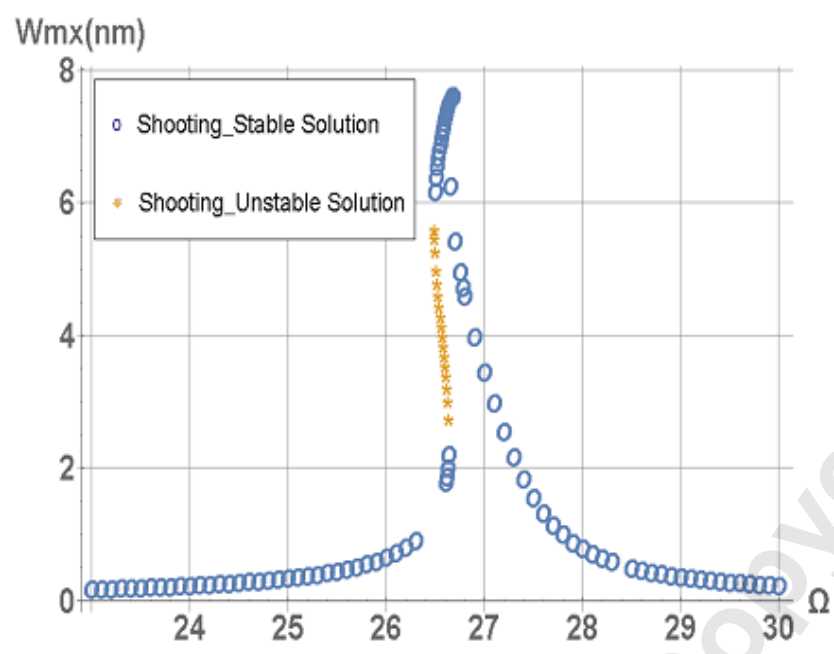

Fig. 6 Frequency-response curves of $5 \mathrm{~nm}$ slacked CNT.

Shown for $V_{\mathrm{DC}}=V_{\mathrm{AC}}=1 \mathrm{~V}$ and a quality factor of 100 .

Next, we consider more cases under large electric loads. As discussed previously, the nonlinear behavior of slacked CNTs under large electrostatic force has not been explored before. Here we conduct such study using the shooting technique and the new efficient model. Consider the same case study of a non-hollow CNT of $L=1000$ $\mathrm{nm}, \tilde{R}=5 \mathrm{~nm}, d=200 \mathrm{~nm}$, Young modulus $E=1 \mathrm{TPa}$, and mass density $\rho=1.3 \mathrm{~g} / \mathrm{cm}^{3}$.

\subsection{Effect of DC voltage}

First, we study the effect of the DC load on the CNT nonlinearity. Fig7 shows that the 5nm slacked CNT exhibits generally softening behavior for small vibration amplitude and hardening behavior for larger oscillation. Comparing Fig. 7a to Fig. 7e shows that increasing DC voltage amplifies the quadratic effect of the electrostatic force, and hence more evident softening effect shows up for the small oscillation. It is worth noting that the initial softening behavior presents in cases a,b, and e is weak in c and does not exist in $\mathrm{d}$. A possible explanation to this is the fact that for small values of $V_{\mathrm{DC}}, \mathrm{a}, \mathrm{b}$, the quadratic curvature nonlinearities is strong enough to cause softening effect. In Fig. c, $V_{\mathrm{DC}}$ causes more stretching in the beam making the cubic geometric nonlinearity to become stronger than the curvature nonlinearity. Increasing $V_{\mathrm{DC}}$ further adds quadratic nonlinearities from the electrostatic force, and hence, enhancing again the quadratic nonlinearities. 
Similar phenomenon has also been observed in MEMS, such as electrically actuated MEMS comb drive resonators utilizing folded suspension beams [28]. The nonlinear instability behavior of the beam, changing from softening type to hardening type, has been reported before in the macro scale, for example in a study of snap-through properties of a nonlinear dynamic buckling response of a clamped-clamped buckled beam [29].

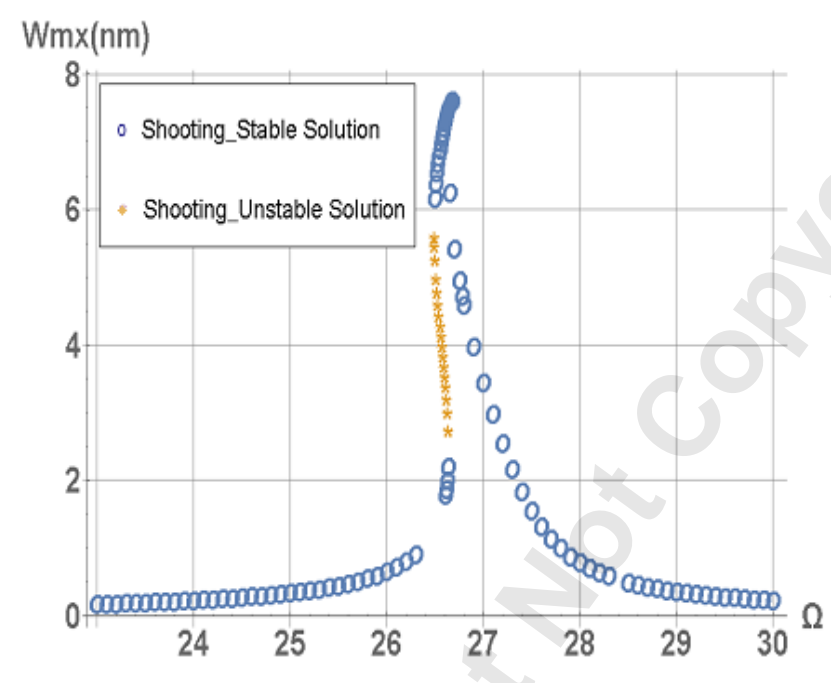

a

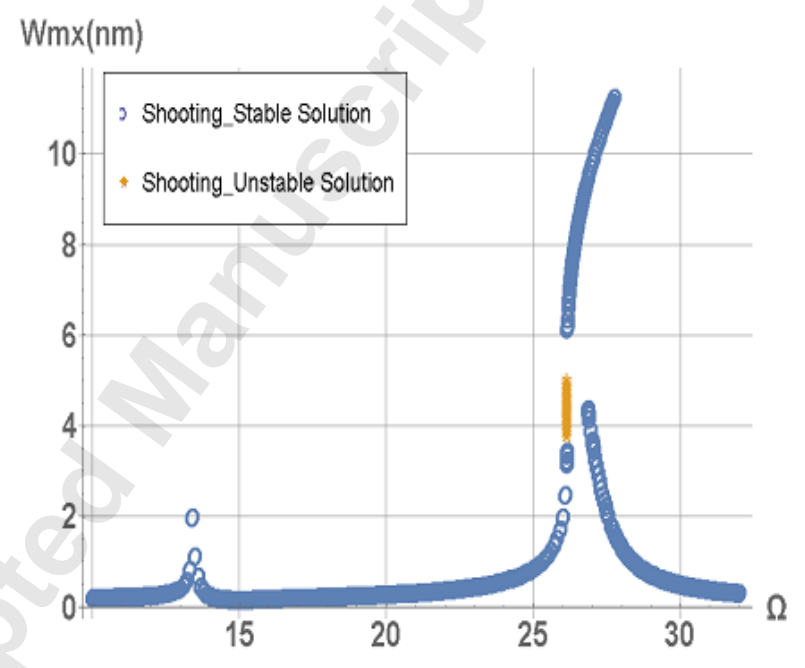

b 


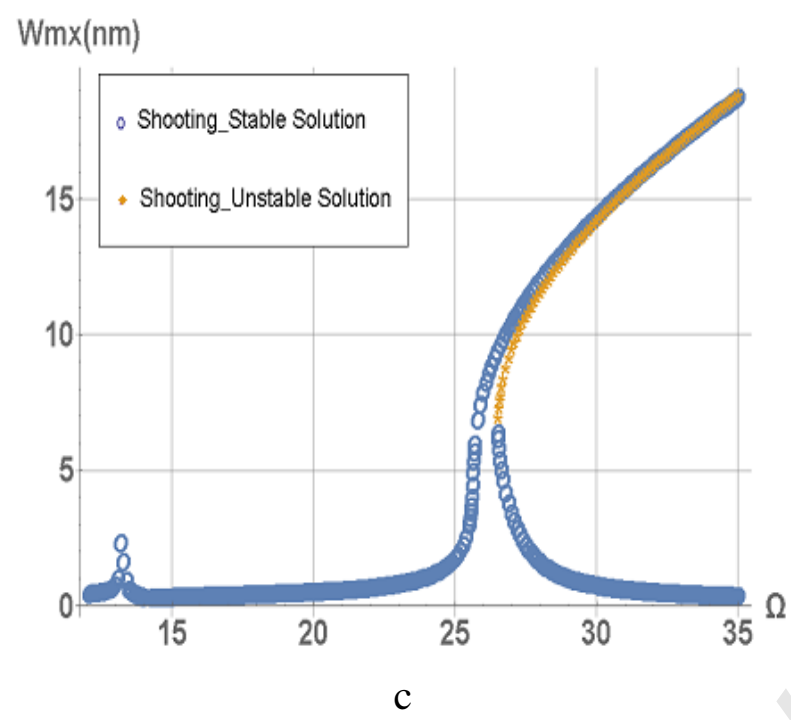

$\mathrm{Wmx}(\mathrm{nm})$

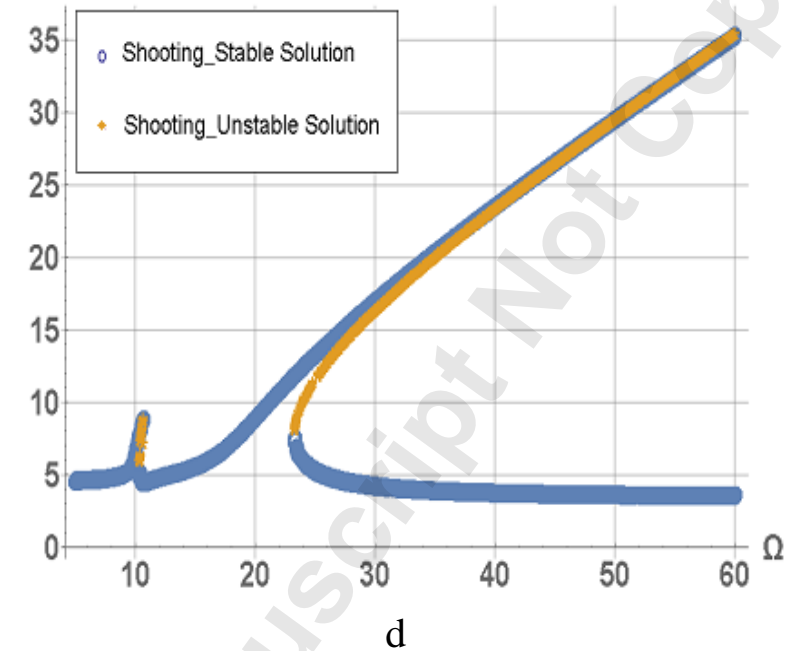

Wmx(nm)

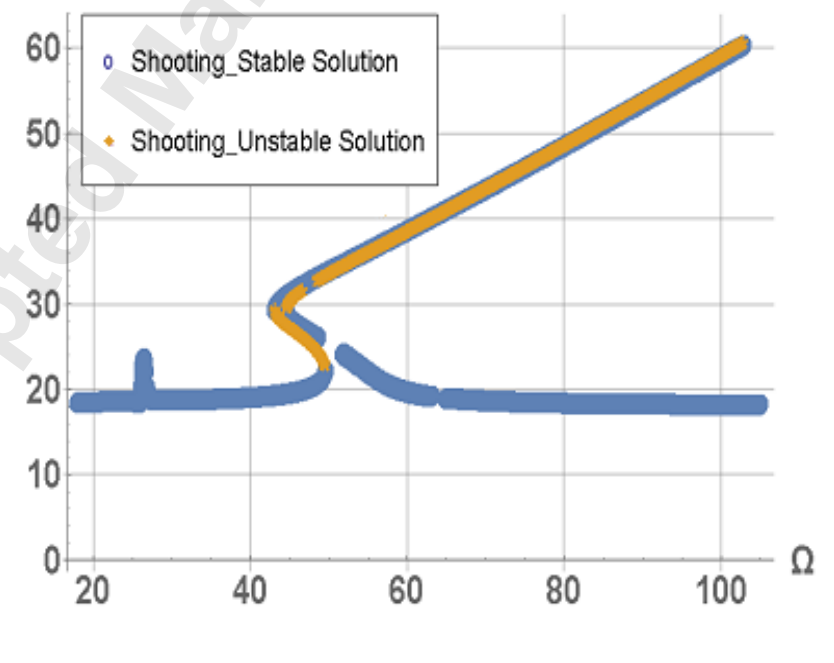

$\mathrm{e}$

Fig. 7 Frequency-response curves for (a) $V_{\mathrm{DC}}=2 \mathrm{~V} V_{\mathrm{AC}}=1 \mathrm{~V}$ (b) $V_{\mathrm{DC}}=3 \mathrm{~V} V_{\mathrm{AC}}=1 \mathrm{~V}$ (c) $V_{\mathrm{DC}}=10 \mathrm{~V} V_{\mathrm{AC}}=1 \mathrm{~V}(\mathbf{d}) V_{\mathrm{DC}}=20 \mathrm{~V}$ $V_{\mathrm{AC}}=1 \mathrm{~V}(\mathbf{e}) V_{\mathrm{DC}}=30 \mathrm{~V} V_{\mathrm{AC}}=1 \mathrm{~V}$. Results are shown for 5nm slacked CNT and a quality factor of 100 . 


\subsection{Effect of AC voltage}

Next, the impact of the AC voltage is studied. Figs8-11 show that larger AC voltage introduces larger superharmonic and sometimes subharmonic resonances, Fig10 (b, c) and Fig11 (b). More impressive, Fig10(c) reveals the coexistence of five solutions including three stable and two unstable ones near $\Omega=38$. Similar results were revealed recently using continuation techniques [22] where only an unknown stable solution of larger amplitude has been obtained. Now we can confirm that the unknown stable solution is part of the superharmonic resonance. We recall here that at superharmonic resonance of order two, the structure responds at twice the excitation frequency while it responds at three times that in the case of superharmonic resonance of order three. Also, at subharmonic resonance of order half and one third, the structure responds at half and one third the excitation frequency, respectively. It is worth mentioning that the possibility of large response in the case of superharmonic and subharmonic resonances can be surprising (in the linear sense) since they occur at $\mathrm{t}$ frequencies, which usually are considered of safely low amplitude.

This complicated nonlinear phenomenon on electorally actuated CNTs has never been studied before. Such coexistence of several solutions, stable and unstable, primary and subharmonic, has been observed in studies of nonlinear dynamics of MEMS, such as electrically actuated imperfect microbeam actuator [30].

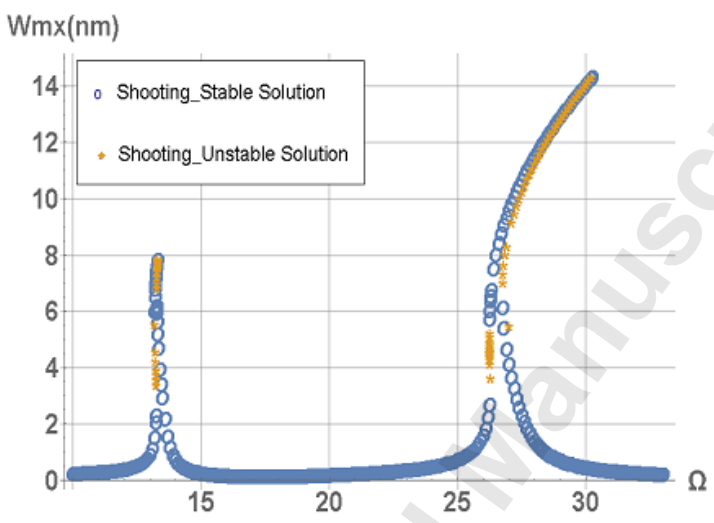

a

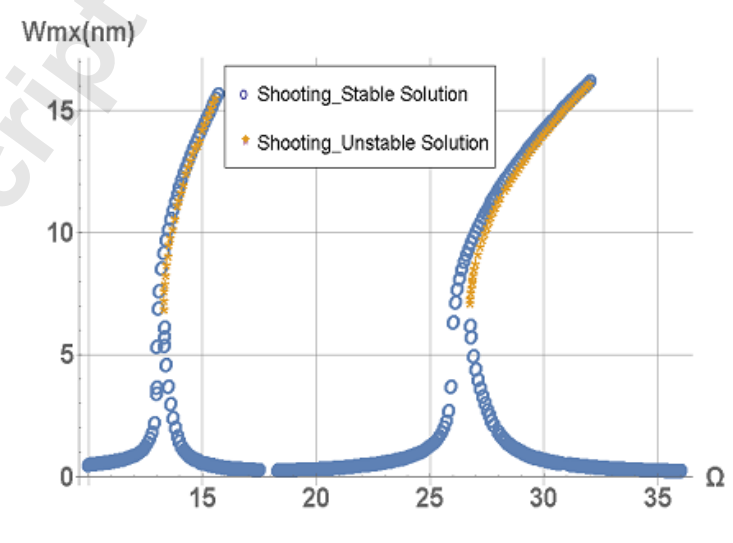

b

Fig. 8 Frequency-response curves for (a) $V_{\mathrm{DC}}=1 \mathrm{~V} V_{\mathrm{AC}}=2 \mathrm{~V}(\mathbf{b}) V_{\mathrm{DC}}=1 \mathrm{~V} V_{\mathrm{AC}}=3 \mathrm{~V}$. Results are shown for $5 \mathrm{~nm}$ slacked CNT and a quality factor of 100 . 


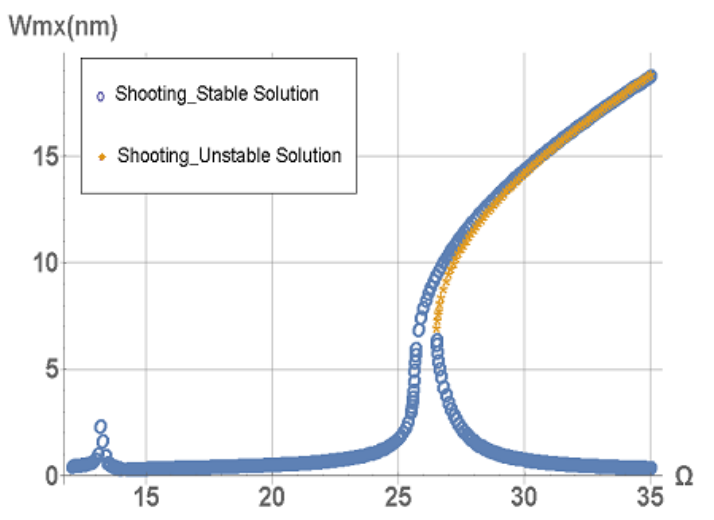

a

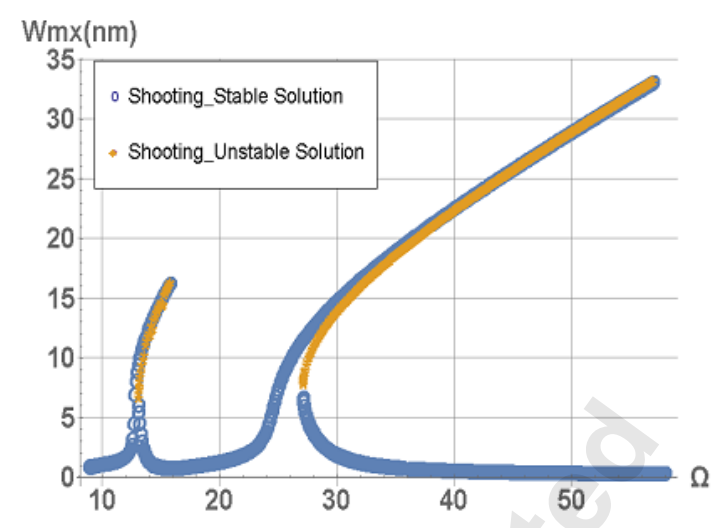

b

Fig. 9 Frequency-response curves for $(\mathbf{a}) \quad V \mathrm{DC}=3 \mathrm{~V} V V_{\mathrm{AC}}=1 \mathrm{~V}(\mathbf{b}) \quad V_{\mathrm{DC}}=3 \mathrm{~V} V V_{\mathrm{AC}}=3 \mathrm{~V}$. Results are shown for $5 \mathrm{~nm}$ slacked CNT and a quality factor of 100

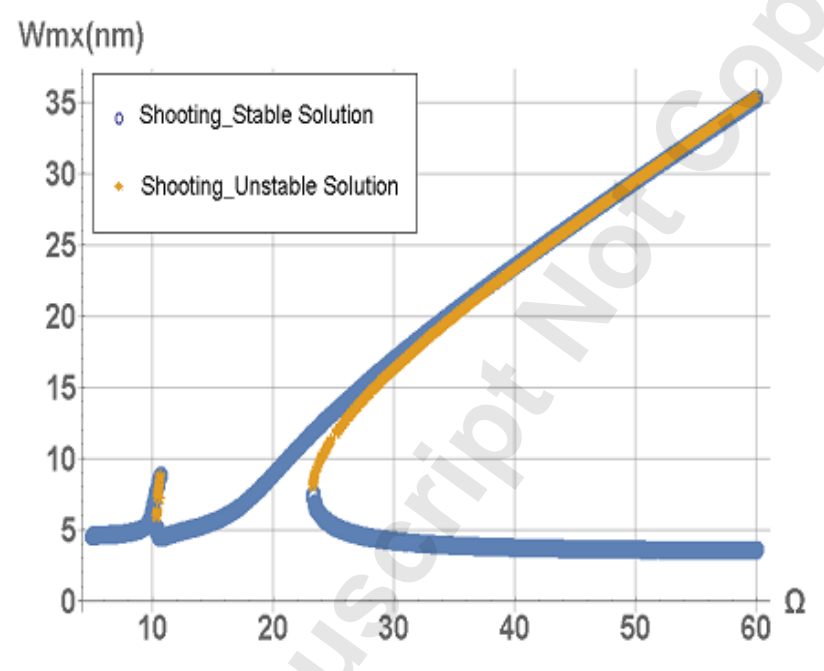

a

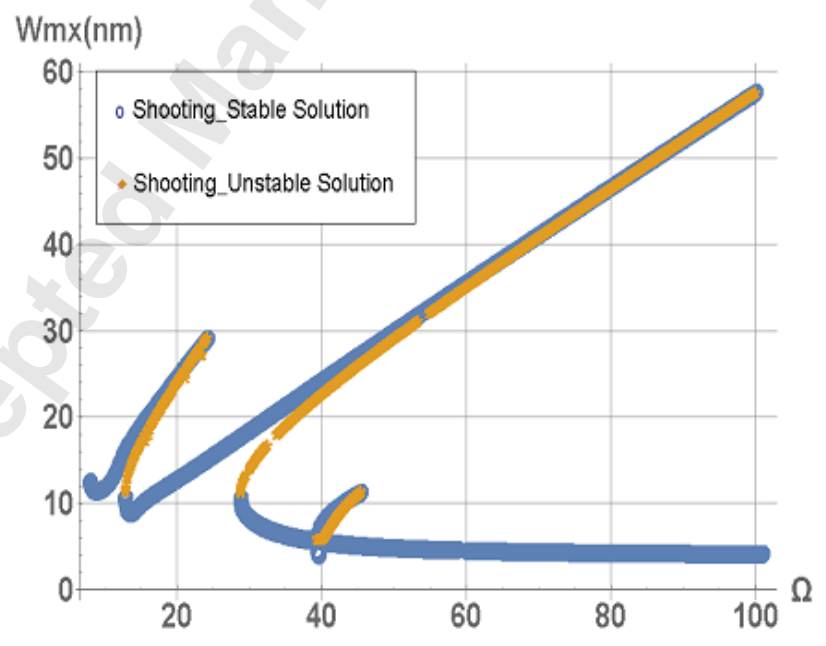

$\mathrm{b}$ 


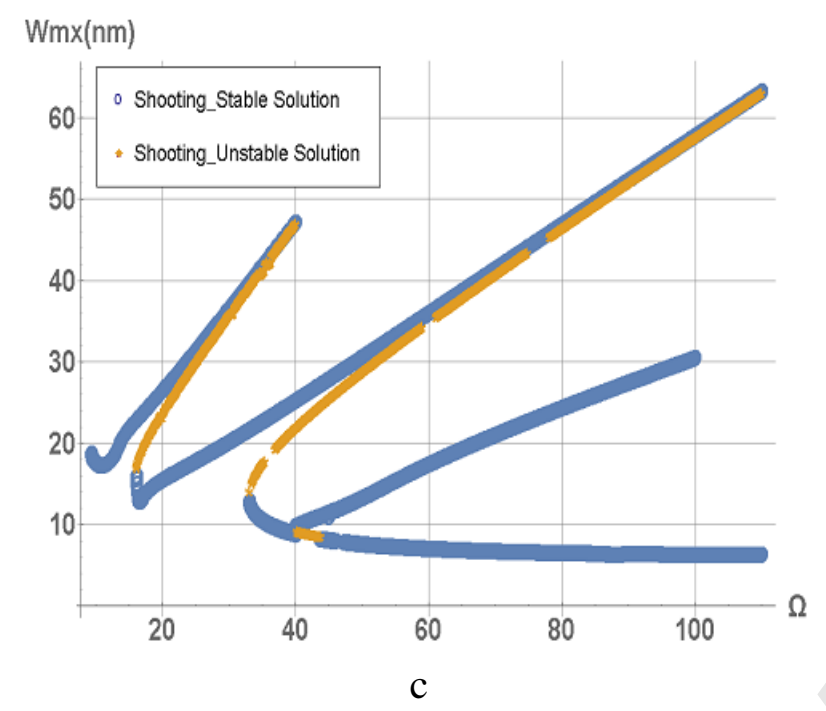

Fig. 10 Frequency-response curves for (a) $V_{\mathrm{DC}}=10 \mathrm{~V} V_{\mathrm{AC}}=1 \mathrm{~V}$ (b) $V_{\mathrm{DC}}=10 \mathrm{~V} V_{\mathrm{AC}}=5 \mathrm{~V}$ (c) $V_{\mathrm{DC}}=10 \mathrm{~V} V_{\mathrm{AC}}=10 \mathrm{~V}$. Results are shown for 5nm slacked CNT and a quality factor of 100 .

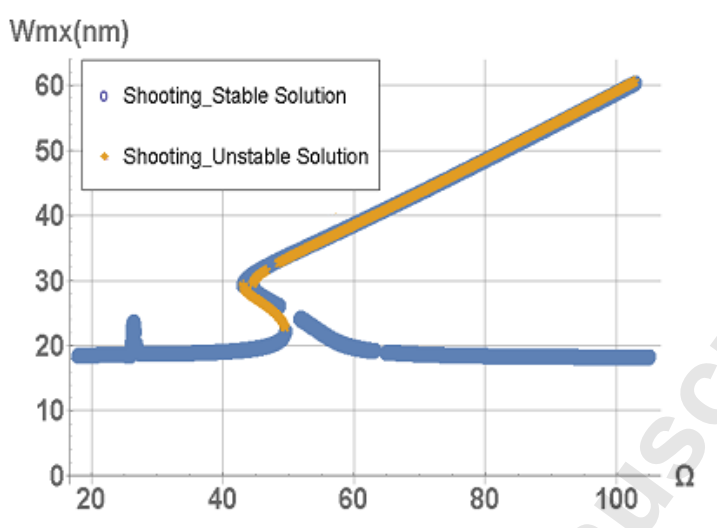

a

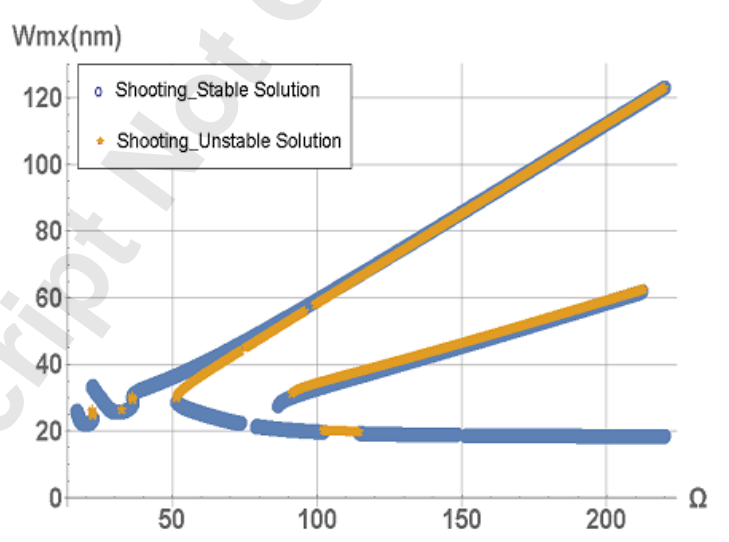

b

Fig. 11 Frequency-response curves for $(\mathbf{a}) V_{\mathrm{DC}}=30 \mathrm{~V} V_{\mathrm{AC}}=1 \mathrm{~V}$ (b) $V_{\mathrm{DC}}=30 \mathrm{~V} V_{\mathrm{AC}}=10 \mathrm{~V}$. Results are shown for $5 \mathrm{~nm}$ slacked CNT and a quality factor of 100 .

From all of these figures above, we can see also that the natural frequency of the system becomes larger when increasing the DC voltage input. Jointly increasing DC and AC voltage would of course enlarge the amplitude of the deflection of the CNT. Moreover this would cause more complicated nonlinear behavior and increase the instability of the CNT by introducing the changing from softening to hardening behavior of the primary resonance and triggering both subharmonic and superharmonic resonance.

\subsection{Effect of initial curvature}

Next we investigate the influence of the slack level on the frequency-response curve of the CNTs. From Fig12, it can be obviously seen that larger slack level increases the quadratic effect, which is shown as softening behavior. It is indeed expected that the system will experience significant softening with larger initial curvatures due to the 
amplification of the quadratic nonlinearity. Comparing Fig12a to Fig12c, it shows that the natural frequency of the system shifts from 20 to 65 when increasing the initial slack level from $5 \mathrm{~nm}$ to $20 \mathrm{~nm}$.
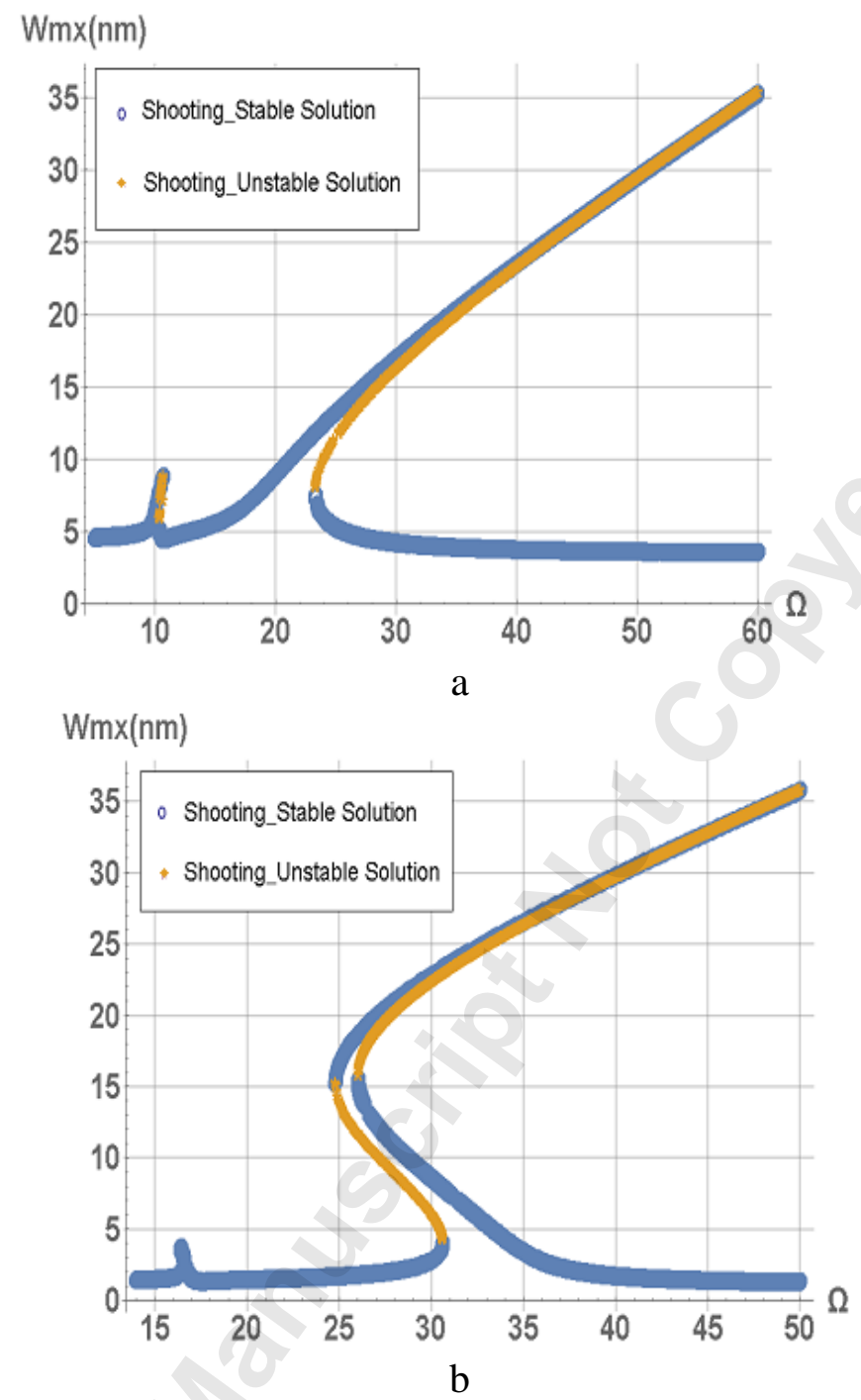

Wmx $(\mathrm{nm})$

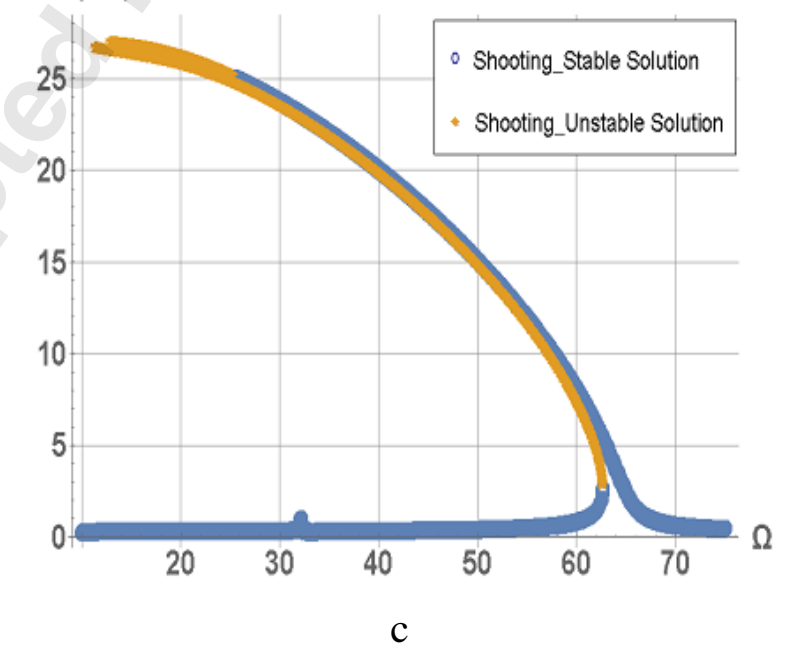

Fig. 12 Frequency-response curves for slack level (a) $5 \mathrm{~nm}(\mathbf{b}) 10 \mathrm{~nm}$ (c) $20 \mathrm{~nm}$ Results are shown for $V_{\mathrm{DC}}=10 \mathrm{~V}, V_{\mathrm{AC}}=1 \mathrm{~V}$ and a quality factor of 100 . 


\subsection{Small diameter and large initial slack case}

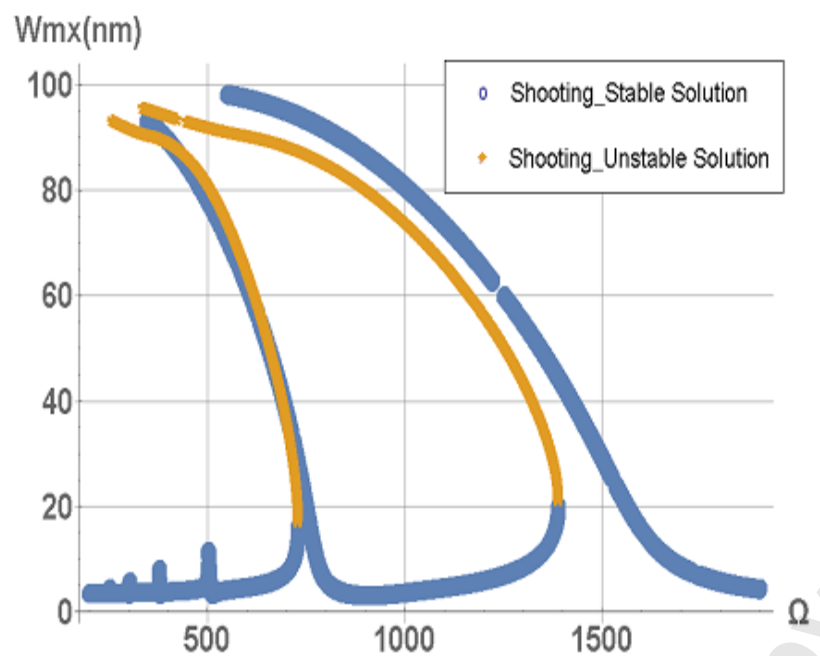

Fig. 13 Frequency-response curves for slack level $100 \mathrm{~nm}$. Results are shown for $V_{\mathrm{DC}}=10 \mathrm{~V}, V_{\mathrm{AC}}=10 \mathrm{~V}$ and a quality factor of 100 .

Finally, we consider another case study of CNTs with typical dimensions in $[11,12]$ for a non-hollow CNT of $L=1750 \mathrm{~nm}, \tilde{R}=1 \mathrm{~nm}, d=500 \mathrm{~nm}$, Young modulus $E=1$

$\mathrm{TPa}$, and mass density $\rho=1.3 \mathrm{~g} / \mathrm{cm}^{3}$ and a slack level of $100 \mathrm{~nm}$. The results are shown in Fig 13, which reveal significant linear natural frequency shift. The primary non-dimensional natural frequency in this case is around 1400, which is much larger than 24 in 5nm slacked CNT case under the same electrostatic force. This significant amplifying of natural frequency might be due to the very small radius. Moreover, for small radius CNT, a tiny slack level will soften the fundamental mode as studied before [27]. The quadratic effect is dominant in this case with really large initial curvature, which is shown as softening behavior of the CNT. Also the co-existence of the primary and superharmonic resonance has been found around $\Omega=750$, which is half of the primary resonance frequency. Also the system is excited by superharmonic resonance of order three around $\Omega=500$ with much smaller amplitude compared to the superharmonic of order two as shown in Fig13. This on the other hand confirms that quadratic nonlinearity is more critical in this case. In addition, some other little peaks have been triggered, which seems to higher order superharmonic resonances introduced by the higher order electrostatic nonlinearities.

Similar investigation on the impact of DC and AC voltage on the nonlinearity has been done for this case. Fig13 to Fig 15 shows that the 100nm slacked CNT exhibits generally softening behavior and would never switch to hardening behavior like the 5nm slacked case. Comparing Fig. 13 with Fig. 14 shows that increasing DC voltage amplifies the quadratic effect of the electrostatic force. The primary resonance even meets the superharmonic in Fig14. 


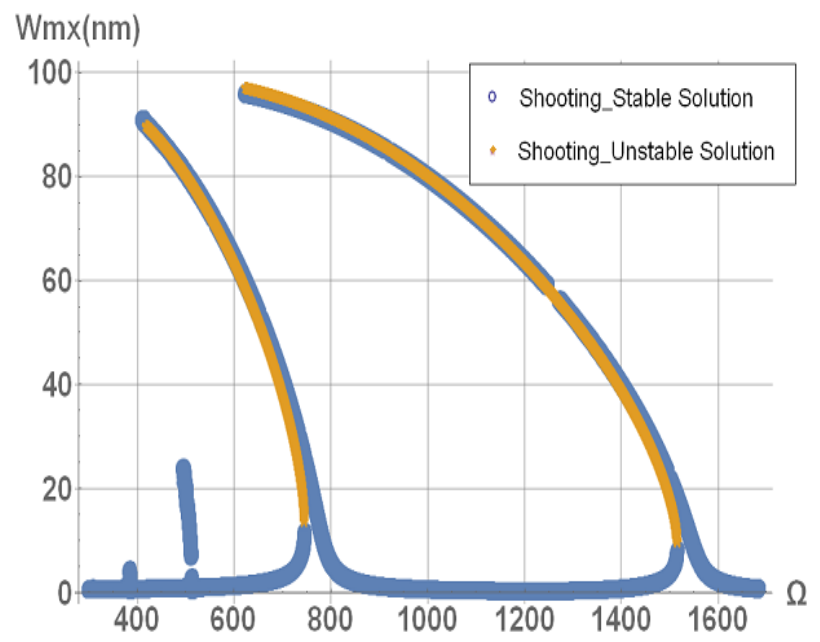

Fig. 14 Frequency-response curves for $V_{\mathrm{DC}}=1 \mathrm{~V} V_{\mathrm{AC}}=10 \mathrm{~V}$. Results are shown for 100nm slacked CNT and a quality factor of 100 .

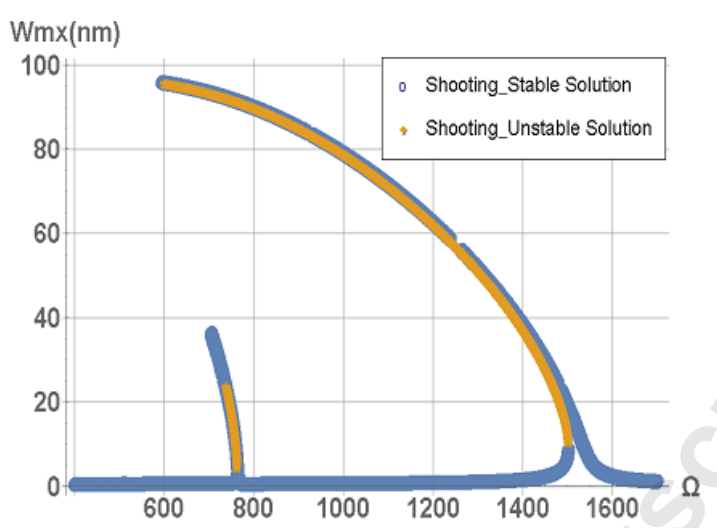

a

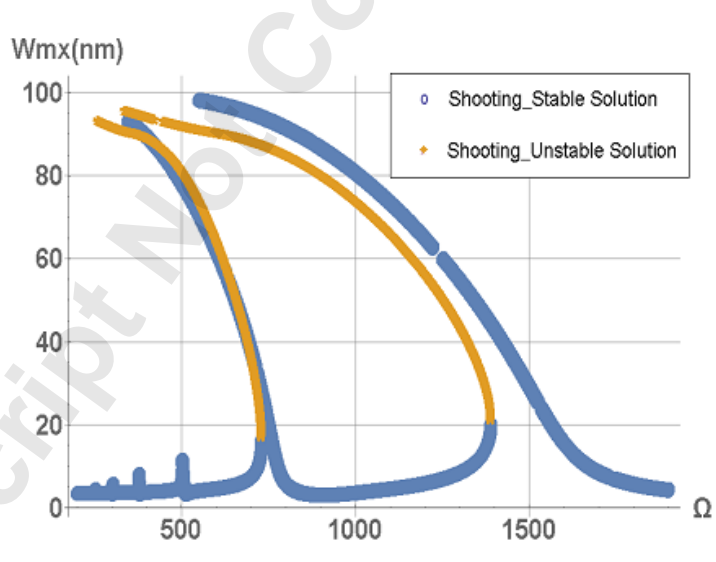

$\mathrm{b}$

Fig. 15 Frequency-response curves for (a) $V_{\mathrm{DC}}=10 \mathrm{~V} V_{\mathrm{AC}}=1 \mathrm{~V}$ (b) $V_{\mathrm{DC}}=10 \mathrm{~V} V_{\mathrm{AC}}=10 \mathrm{~V}$. Results are shown for 100nm slacked CNT and a quality factor of 100 .

From Fig15, it can be seen that larger AC voltage amplifies the superharmonic resonance of order two.

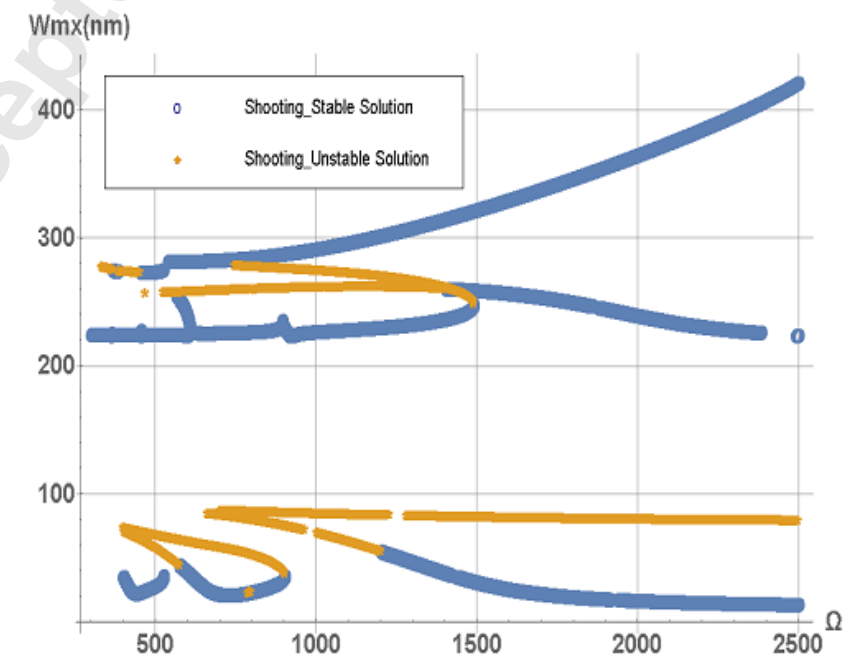


Fig. 16 Frequency-response curves for $V_{\mathrm{DC}}=30 \mathrm{~V} V_{\mathrm{AC}}=10 \mathrm{~V}$. Results are shown for 100nm slacked CNT and a quality factor of 100 .

Increasing DC voltage further leads to the interesting results in Fig. 16. New upper solutions appear corresponding to the vibration at the other well (other buckled or curved position). This result indicates the richness of the dynamics and the possibility to discover more solutions at the high vibration amplitude in addition to what have been demonstrated in this paper.

\section{Conclusions}

In this work, an investigation into the dynamic behavior of an electrically actuated slacked single-walled CNT when actuated by a DC force and an AC harmonic load was presented. Taylor expansion has been used to substitute the complicated electrostatic force term. It has been proven that this substitution could make the numerical simulation process much more efficient and would not affect the accuracy of the result.

Moreover, taking advantage of shooting technique and the substitution of Taylor expansion gives us a chance to generate results under wider range of electrostatic force load. We found that there are some unique and interesting nonlinear behaviors of CNTs when actuated by larger electrostatic force, which have never been studied before on CNTs. Large DC voltage can amplify the quadratic nonlinearity. However the CNTs are quite strong they might return to hardening behavior for some range. Increasing AC voltage will introduce large superharmonic resonance. The superharmonic resonance could even coexist with the primary resonance. Increasing the input voltage would also increase a chance to excite the subharmonic resonance, which is very important from the stability point of view and might be completely overlooked in the design of CNT devices and resonators. Larger slack level increases the quadratic effect, which is shown as softening behavior and superharmonic resonance of order two. This modeling approach enables parameter studies and design optimizations for CNTs applications with respect to nonlinear dynamic behavior.

\section{References}

[1] Pelesko, J. A. and Bernstein, D.H., "Modeling MEMS and NEMS, Chapman \& Hall/CRC Press", 2003.

[2] Gibson, R. F., Ayorinde, E. O., and Wen, Y. F., "Vibrations of Carbon Nanotubes and Their Composites: A Review,” Compos. Sci. Technol., 67, 1-28, 2008. 
[3] Rhoads, J. F., Shaw S. W., Turner, K. L., "Nonlinear Dynamics and Its Applications in Micro- and Nanoresonators,” J. Dynamic Systems, Measurement, and Control, 132, 034001-1,2010.

[4] Lifshitz, R., and Cross, M. C., "Nonlinear Dynamics of Nanomechanical and Micromechanical Resonators,” Review of Nonlinear Dynamics and Complexity, H. G. Schuster, 1-52, 2008.

[5] Craighead, H. G., “Nanoelectromechanical Systems”, Science, 290, 1532-1535,2000.

[6]Antonio Pantano, Mary C. Boyce, and David M. Parks, "Nonlinear Structural Mechanics Base Modeling of Carbon Nanotube Deformation”, Physical Review Letters, Vol.91, 14.145504, 2003.

[7] Postma, H., Kozinsky, I., Husain, A., Roukes,M., "Dynamic range of nanotube- and nanowire-based electromechanical systems”, Appl. Phys. Lett. 86, 223105, 2005.

[8] Dequesnes, M., Tang, S., Aluru, N.R., "Static and dynamic analysis of carbon nanotube-based switches”, J. Eng. Mater. Technol, 126, 230-237, 2004.

[9]W. G. Conley, A. Raman, C.M. Krousgrill and S. Mohammadi, "Nonlinear and nonplanar dynamics of suspended nanotube and nanowire resonators”, Nano Lett., 8, 1590-1595, 2008.

[10] Gibson, R.F., Ayorinde, E.O., Wen, Y.F., "Vibrations of carbonnanotubes and their composites: a review”, J. Comput Sci. Technol. 67, 1-28, 2007.

[11] Sazonova, V., Yaish, Y., Üstünel, H., Roundy, D., Arias. T.A.,McEuen, P.L., “A tunable carbon nanotubes electromechanical oscillator”, Nature 431, 284-287,2004.

[12] Sazonova, V.A., “A tunable carbon nanotube resonator” Ph.D. Thesis, Department of Physics, Cornell University, 2006.

[13] Üstünel, H., Roundy, D., Arias, T.A., "Modeling a suspended nanotube oscillator”, Nano Lett. 5, $523-526,2005$.

[14] Garcia-Sanchez, D., San Paulo, A., Esplandiu, M.J., PerezMurano, F., Forrò, L., Aguasca, A., Bachtold, A, "Mechanical detection of carbon nanotube resonator vibrations", Phys. Rev. Lett. 99, $085501,2007$.

[15] Mayoof, F.N., Hawwa, M.A., "Chaotic behavior of a curved carbon nanotube under harmonic excitation", Chaos Solitons Fractals 42, 1860-1867, 2009.

[16] Ke, C.H., Espinosa, H.D., Pugno, N., "Numerical analysis of nanotube-based NEMS devices_-part II: role of finite kinematics, stretching and charge concentrations”, J. Appl. Mech. 72, 726-731, 2005. 
[17]Yakobson, B., Brabec, C., Bernholc, J., "Nanomechanics of carbon tubes: instabilities beyond linear response", Phys. Rev. Lett, 76, 2511-2514, 1996.

[18] Harik, V.M., "Ranges of applicability of the continuum beam model in the mechanics of carbon nanotubes and nanorods", Solid State Commun, 120, 331-335, 2001.

[19] Younis, M.I., Abdel-Rahman, E.M., Nayfeh, A.H., “A reduced-order model for electrically actuated microbeam-based MEMS”, J. Microelectromech. Syst, 12, 672-680, 2003.

[20] Younis M. I., "MEMS Linear and Nonlinear Statics and Dynamics”, Springer, 2011.

[21] Ruzziconi, L., Younis, M. I., and S. Lenci, "Multistability in an electrically actuated carbon nanotube: a dynamical integrity perspective,” Nonlinear Dynamics, DOI 10.1007/s11071-013-0986-5, Vol. 74, 533-549, 2013.

[22] C.C. Wu and Z. Zhong, "Capacitive spring softening in single-salled carbon nanotube nanoelectromechanical resonators", Nano Lett, 11, 1448-1451, 2011.

[23] L. Jin, J. Mei and L. Li, "Nonlinear dynamics of a doubly clamped carbon nanotube resonator considering surface stress", RSC Advances, 5,7251, 2015.

[24] Gomez A. Castellanos, Meerwaldt H. B, Venstra W. J., Zant H. S.J.van der, and Steele G. A. Steele, "Strong and tunable mode coupling in carbon nanotube resonators," Physical Review B 86,041402(R), 2012.

[25] Ouakad, H. and Younis, M. I, "Nonlinear Dynamics of Electrically Actuated Carbon Nanotube Resonators", J. of Comp. and Non. Dyn., Vol. 5, 011009, 13, 2010.

[26] Ouakad, H. and Younis, M. I, "Natural Frequencies and Mode Shapes of Initially Curved Carbon Nanotube Resonators under Electric Excitation”, J. of Sound and Vibrations, Vol. 330, 3182-3195, 2011.

[27] Ouakad, H. and Younis, M. I, "Dynamic Response of Slacked Carbon Nanotube Resonators”, Non. Dyn., 67:1419-1436, DOI 10.1007/s11071-011-0078-3, 2012.

[28] A. M, Elshurafa, K. Khirallah and H. H. Tawfil, "Nonlinear Dynamics of Spring Softening and Hardening in Folded-MEMS Comb Drive Resonators”, Journal of Microelectromechanical Systems, Vol 20, No.4, 2011.

[29] W Y Poon, C F Ng and Y Y Lee, "Dynamic Stability of Curved Beam under Sinusoidal Loading”, Jounal of Aerospace Engineering, Vol. 216, No.4 209-217, 2002.

[30] L. Ruzziconi, A. M Bataineh, M. I Younis, W. Cui and S. Lenci, "Nonlinear Dynamics of An Electrically Actuated Imperfect Microbeam Resonator: Experimental Investigation and Reduced-Order Modeling”, J. Micromech. Microeng. 23, 075012, 2013. 


\section{List of Figure Captions}

Fig.1 Schematic of an initially curved carbon nanotube.

Fig.2 Comparison of the Taylor series expansion and the exact form of the nondimensional electrostatic force.

Fig.3 Percentage error of the Taylor series expansion of the nondimensional electrostatic force.

Fig. 4 Frequency-response curves of (a) $2 \mathrm{~nm}$ and (b) $5 \mathrm{~nm}$ slacked CNT showing hardening and softening behaviors, respectively. Results are shown for $V_{\mathrm{DC}}=V_{\mathrm{AC}}=1 \mathrm{~V}$ and a quality factor of 100 .

Fig.5. Frequency response curve of $2 \mathrm{~nm}$ slacked CNT. Shown for $V_{\mathrm{DC}}=V_{\mathrm{AC}}=1 \mathrm{~V}$ and a quality factor of 100 .

Fig. 6 Frequency-response curves of $5 \mathrm{~nm}$ slacked CNT. Shown for $V_{\mathrm{DC}}=V_{\mathrm{AC}}=1 \mathrm{~V}$ and a quality factor of 100 .

Fig. 7 Frequency-response curves for (a) $V_{\mathrm{DC}}=2 \mathrm{~V} V_{\mathrm{AC}}=1 \mathrm{~V}$ (b) $V \mathrm{DC}=3 \mathrm{~V} V_{\mathrm{AC}}=1 \mathrm{~V}$ (c) $V_{\mathrm{DC}}=10 \mathrm{~V}$ $V_{\mathrm{AC}}=1 \mathrm{~V}(\mathbf{d}) V_{\mathrm{DC}}=20 \mathrm{~V} V_{\mathrm{AC}}=1 \mathrm{~V}(\mathbf{e}) V_{\mathrm{DC}}=30 \mathrm{~V} V_{\mathrm{AC}}=1 \mathrm{~V}$. Results are shown for $5 \mathrm{~nm}$ slacked CNT and a quality factor of 100 .

Fig. 8 Frequency-response curves for (a) $V_{\mathrm{DC}}=1 \mathrm{~V} V_{\mathrm{AC}}=2 \mathrm{~V}$ (b) $V_{\mathrm{DC}}=1 \mathrm{~V} V_{\mathrm{AC}}=3 \mathrm{~V}$. Results are shown for 5nm slacked CNT and a quality factor of 100 .

Fig. 9 Frequency-response curves for (a) $V D C=3 \mathrm{~V} V A C=1 \mathrm{~V}(\mathbf{b}) V D C=3 \mathrm{~V} V A C=3 \mathrm{~V}$. Results are shown for $5 \mathrm{~nm}$ slacked CNT and a quality factor of 100

Fig. 10 Frequency-response curves for (a) $V_{\mathrm{DC}}=10 \mathrm{~V} V_{\mathrm{AC}}=1 \mathrm{~V}$ (b) $V_{\mathrm{DC}}=10 \mathrm{~V} V_{\mathrm{AC}}=5 \mathrm{~V}$ (c) $V_{\mathrm{DC}}=10 \mathrm{~V}$ $V_{\mathrm{AC}}=10 \mathrm{~V}$. Results are shown for 5nm slacked CNT and a quality factor of 100 .

Fig. 11 Frequency-response curves for $(\mathbf{a}) V_{\mathrm{DC}}=30 \mathrm{~V} V_{\mathrm{AC}}=1 \mathrm{~V}(\mathbf{b}) V_{\mathrm{DC}}=30 \mathrm{~V} V_{\mathrm{AC}}=10 \mathrm{~V}$. Results are shown for $5 \mathrm{~nm}$ slacked CNT and a quality factor of 100 .

Fig. 12 Frequency-response curves for slack level (a)5nm (b)10nm (c)20nm Results are shown for $V_{\mathrm{DC}}=10 \mathrm{~V}, V_{\mathrm{AC}}=1 \mathrm{~V}$ and a quality factor of 100 .

Fig. 13 Frequency-response curves for slack level 100nm. Results are shown for $V_{\mathrm{DC}}=10 \mathrm{~V}, V_{\mathrm{AC}}=10 \mathrm{~V}$ and a quality factor of 100 .

Fig. 14 Frequency-response curves for $V_{\mathrm{DC}}=1 \mathrm{~V} V_{\mathrm{AC}}=10 \mathrm{~V}$. Results are shown for 100nm slacked CNT and a quality factor of 100 .

Fig. 15 Frequency-response curves for (a) $V_{\mathrm{DC}}=10 \mathrm{~V} V_{\mathrm{AC}}=1 \mathrm{~V}$ (b) $V_{\mathrm{DC}}=10 \mathrm{~V} V_{\mathrm{AC}}=10 \mathrm{~V}$. Results are shown for $100 \mathrm{~nm}$ slacked CNT and a quality factor of 100 .

Fig. 16 Frequency-response curves for $V_{\mathrm{DC}}=30 \mathrm{~V} V_{\mathrm{AC}}=10 \mathrm{~V}$. Results are shown for 100nm slacked CNT and a quality factor of 100 . 
